\title{
Impact of Ultra Sound Therapy on Myofascial Pain Dysfunction Syndrome along with Masticatory Muscles
}

\author{
Shweta RK ${ }^{1 *}$, Prashant KP1 ${ }^{1}$, Siddharth $\mathrm{S}^{2}$ and Abhay $\mathrm{D}^{1}$ \\ ${ }^{1}$ Department of Oral and Maxillofacial Surgery, Government Dental College and \\ Hospital, Nagpur,India \\ 2Department of Orthodontics, Mansarovar Dental College \& Reseach, Bhopal Madhya \\ Pradesh, India
}

\section{Research Article}

Volume 2 Issue 2

Received Date: April 13, 2018

Published Date: May 26, 2018

*Corresponding author: Shweta R Kamble, Associate Professor, Department of Oral and Maxillofacial Surgery, Government Dental College and Hospital, Nagpur,India, Email:kamble_shweta@yahoo.com

\section{Abstract}

Myofascial pain dysfunction syndrome is dysfunction of the masticatory apparatus related to spasm of the muscles of mastication. For evaluating the role of ultrasound therapy in Myofascial Pain with muscles of mastication, 31 study groups and 31 control group given ultrasound therapy for consecutive 15 days. Electomyographic study was done to evaluate the function of muscles of mastication before and after giving Ultrasound therapy. Ultrasound assumed to have thermal and mechanical effect on the target tissue resulting in an increased local metabolism, circulation, extensibility of connective tissue and tissue regeneration, thus decrease joint stiffness, provide pain relief improve mobility and reduce muscle spasm. Thus safety ultrasonic therapy when properly employed has beneficial effect for alleviation of Myofascial pain the purpose for which the study has been carried out. Study proved that Ultrasound therapy has profound effect in Myofascial Pain Dysfunction syndrome to reduce muscle tension, spasm and improving jaw function.

Keywords: Ultrasound; electomyographic activity; Myofascial Pain Dysfunction Syndrome

\section{Introduction}

Myofascial Pain Dysfunction syndrome is a psychophysiologic disease primarily involves muscles of mastication [1]. Myofascial pain syndrome is defined as pain disorder involving pain referred from trigger points within myofascial structures, either in local or distant from the pain. A trigger point is defined as a localized tender area in a firm band of skeletal muscle, tendon or ligament .These point can occur in any skeletal muscle of body mostly in the head, neck shoulders and lower back MPDS has been characterized by constant dull, pre auricular pain which may be radiating and diffuse in nature. It is frequently associated with painful or limited movement and clinking. Schwartz \& Chayes [2] considered emotional factors cause of the syndrome. Campbell $[1,3]$ study on patient concluded that pain could be induced in muscles by vasoconstriction, cold, anoxia, acidosis or fatigue. Blood supply therefore a factor and necessary to meet the metabolic requirement of muscles 


\section{International Journal of Surgery \& Surgical Techniques}

and this could be disturbed by tension. Travell $[3,4]$ suggested that skeletal muscle stress could be a cause of these symptoms initiated by chilling, trauma or fatigue. Laskin [5] pointed out that symptoms of Mandibular dysfunctions were usually associated with fatigue, spasm or both in masticatory muscle. Thomson [3] concluded altered or excessive muscle activity to be likely causative factor to cause this syndrome. Factors contributing to initiation of MPDS include muscular hyper function, physical disorder, injuries to tissue Para functional habit, disuse, nutritional problem, psychological stress and sleep disturbance. Although MPDS usually starts as functional disorder [6] it ultimately can lead to organic changes in the joints or muscles of mastication and alternation in occlusion.

Patient suffering from MPDS often need a multidisciplinary approach and long term follow up for satisfactory management Therapeutic ultrasound is one of the treatment modalities for MPDS. Ultrasound assumed to have thermal and mechanical effect $[7,8]$ on the target tissue resulting in an increased local metabolism, circulation, extensibility of connective tissue and tissue regeneration, thus decrease joint stiffness, provide pain relief improve mobility and reduce muscle spasm. The safety ultrasonic therapy when properly employed has beneficial effect for alleviation of Myofascial pain the purpose for which the study has been carried out.

\section{Materials and Methods}

This study was carried out in Department of Oral and Maxillofacial Surgery at Government Dental Collage and Hospital Nagpur. Detailed screening of the 10,000 patient who reported with Out Patient Department (OPD) was done. Patient with different age groups above 15-16 years, pain to specific region as mild moderate or intense during movement, or at rest, tenderness over masseter, temporalis muscle, lateral pterygoid and medial pterygoid unilaterally or bilaterally, pain in the temporomandibular joint region on at least one of the joint involved, hypermobility of the joint, difficulty in mouth opening after surgical intervention (such as extraction) were included in the study. While Patient with different age groups above 15-16 years, normal mouth opening, hypermobility of joint without pain were included in control group. Patient with systemic diseases like cardiac diseases, acute infection, young age group where epiphysis of growing bone is present, acute history of external trauma trigeminal neuralgia, maxillary sinusitis, migraine, thrombophlebitis or phlethrombosis or region of inadequate circulation, neoplasm, ankylosis were excluded from study.

The physio-sound plus ultrasound unit Figure 1 There is a source of high frequency current [9] which is conveyed by a coaxial cable to a transducer circuit or treatment head. Inside transducer circuit via a linking electrode. The crystal (quartz / a barium titanate crystal) which deform when subjected to a varying potential difference being fused to the metal front plate of the treatment head. Any change in shape of crystal causes a movement of the metal front plate which in turn produces an ultrasonic wave. These sound waves oscillate at a rate of 1,000,000 times per seconds.

The physio-sound plus ultrasound unit [5] is used to provide continuous ultrasound waves at frequency of 1 $\mathrm{MHz}$, a pulse repetition rate $120 \mathrm{MHz}$ and an intensity of 0.75 to 3 watt $/ \mathrm{cm}^{2}$. The sound head was placed on patient's skin over the affected area 'Aquasonic gel' was applied to keep the sound head in firm contact while it moved over the patient's skin widely used during giving therapeutic ultrasound causing $72.6 \%$ transmission of waves. Time is determined by the size of the area to be treated. It takes approximately 1 minute per 4 square inches of area. Ultrasound therapy was given to the patient and also to the control group for 15 days. The duration for each therapy was for 10 minutes. The intensity of the ultrasound therapy was starts at low intensity as per symptoms present. The rate of increase of intensity was by 0.1 watt $/ \mathrm{cm}^{2}$ per day for a patient over a period of 15 days. The intensity which gave the patient pain relief was determined as per the $100 \mathrm{~mm}$ Visual Analog Scale [10] where one end was labeled no pain and other unbearable pain.

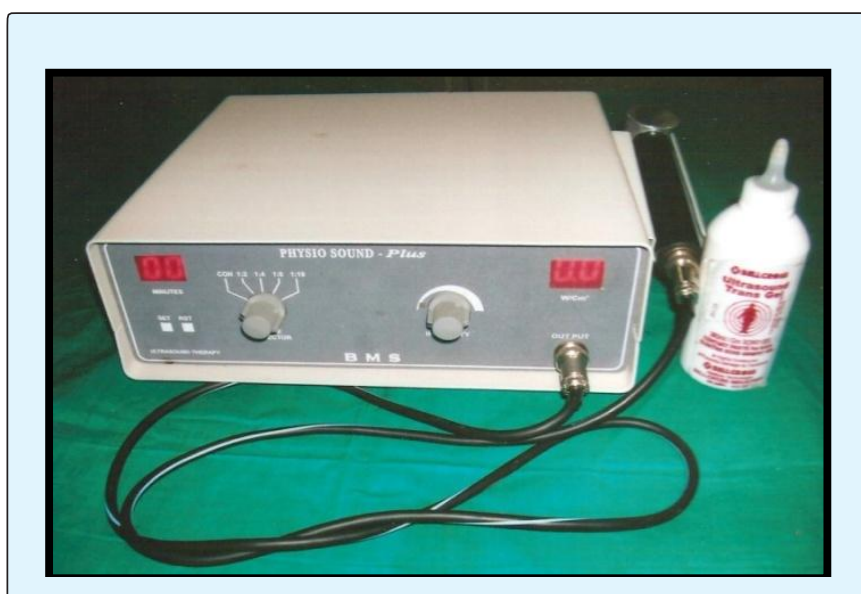

Figure 1: The Physio-sound Plus Ultrasound Unit. 
The basic component of Elecromyographic apparatus is represented in Figure 2. Basic potential are picked up by either a needle electrode or surface electrode and then amplified by amplifier. The output of amplifier is connected to different monitoring devices a) An oscilloscope to permit immediate display and visual monitoring of the potentials, b) An audio amplifier and speaker, to allow acoustic monitoring of the potential and c) A recorder- to make a permanent record of the displayed potentials. The recorder may be one of the several available magnetic tape, film or fiber optic photographic paper readout devices.

Electromyography [9] is only reliable method available for the objective recording of a Patient's muscular function. In clinical dentistry EMG is used primarily to evaluate patients with complaint of temporomandibular joint dysfunction, jaw muscle pain/dysfunction EMG study was done to evaluate changes takes place in muscle of mastication before and after giving ultrasound as therapeutic measure. EMG recording directly measures muscle activity.

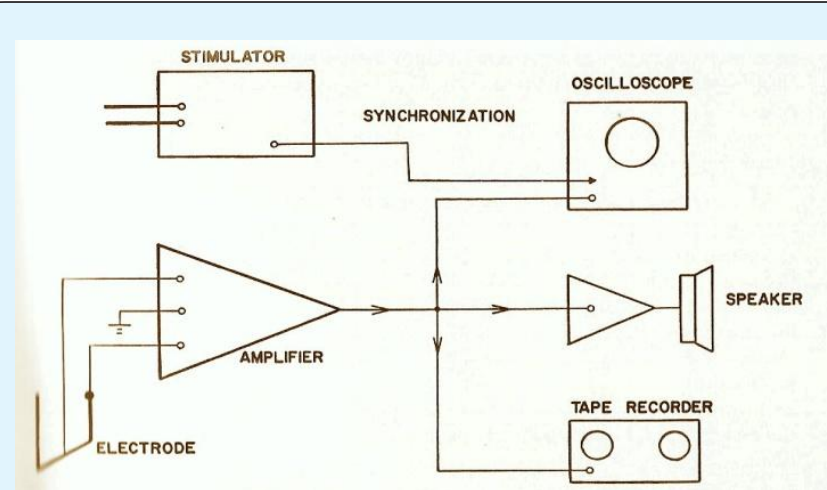

Figure 2: Block diagram showing basic components of typical electromyography.

It can detect muscle hyperactivity and hypoactivity spasm, fatigue and muscle imbalance. Electromyography refers to recording of action potentials of muscle fibers firing singly or in groups near the needle electrode in a muscle. Electromyography examination includes recording of electric activity of voluntary muscles at rest and during volitional activity. EMG examinations are not laboratory test but rather an extension of the clinical examination.

Objective of recording is to obtain a faithful reproduction of the physiologic events that is the wave form picked up by the electrode should be amplified and presented to the observer without distortion and free from interference which may obscure the signal. The recordings were made on RMS EMG EP machine with concentric needle electrodes. A single channel was used since only one muscle was studied at a time which simplifies the analysis of each muscle in terms of frequency, duration amplitude As the electrode penetrate into the muscle, fibers are mechanically stimulated as well as cut and injured giving rise to spontaneous burst of potentials. Spontaneous activity usually refers to potentials recorded after insertion activity has subsided.

\section{Position of the Patient during Procedure}

Temporalis: The EMG of masseter can be carried out either in supine position or sits. The needle is inserted 2.5 $\mathrm{cm}$ posterior to the lateral margin of orbit and above the upper border of zygomatic arch. The muscle is relaxed by slightly opening the mouth and activated by clenching the teeth.

Masseter: The EMG of masseter can be carried out either in supine or sitting position. While the mouth is slightly open. The needle is inserted $2.5 \mathrm{~cm}$ above and in front of the angle of mandible. The muscle can be activated by clenching the teeth.

Lateral pterygoid: The patient lies supine and the needle is inserted $1.5 \mathrm{~cm}$ anterior to the angle of mandible in sagittal plain. The muscle is activated by clenching the teeth.

In this study the examination of masseter, temporalis and lateral pterygoid done during pre and post UST period and the changes observed after examinations were recorded.

Grading given according to activity obtained as:

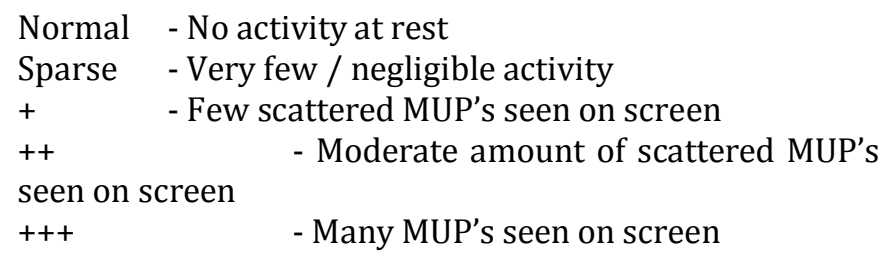

\section{Results}

According to muscular involvement masseter muscle involvement was seen in $93.5 \%$, temporalis muscle involvement seen in $80.6 \%$, pterygoid muscle involvement seen in $19.4 \%$, and muscle involvement of 
posterior cervical group of muscle was seen in $3.2 \%$ of patients as shown in Figure 3. In control group all patients were asymptomatic. They did not show any muscular trigger zone involvement.

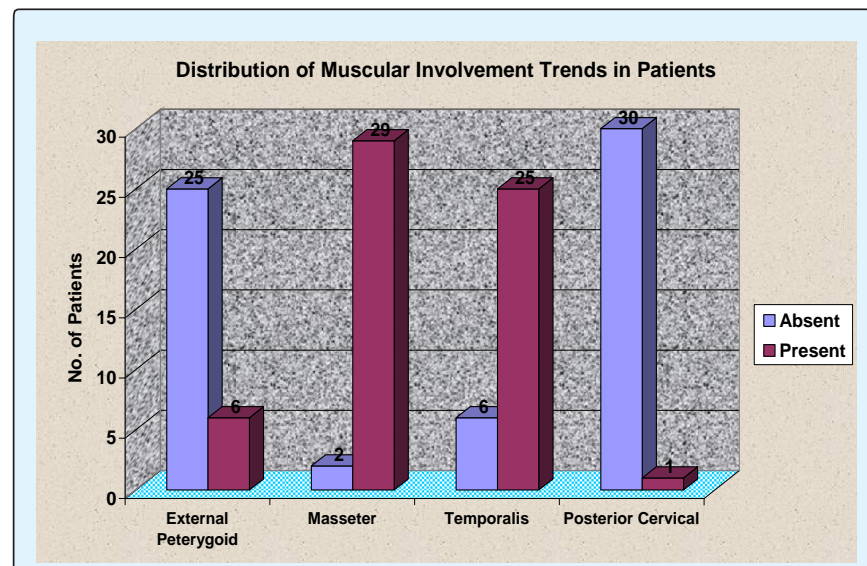

Figure 3: Distribution of muscular involvement trends in patients.

And greatest incidence found in female at age group 21-30 (33.3\%) and in male patient greatest incidence found at age group 31-40 (30.8\%). Myofascial pain dysfunction syndrome observed more common in Housewives $29 \%$ Followed by students $25.8 \%$ and teachers $9.7 \%$.

After psychological assessment out of total 31 patients $12.9 \%$ showed positive psychological status contributing to disease state. It was observed that $15.4 \%$, psychologic factor with medical condition was present in 1 female patient (5.6\%), and mood disorder with anxiety was present in the 1 male patient (5.6\%). In control group $11.1 \%$ observed having anxiety disorder while rests of all were psychologically healthy they did not show any positive psychological status. In study group a pretherapeutic mouth opening in 31 patients had mean of $27.6129+8.1514 \mathrm{~mm}$, after therapy of ultrasound showed increased in mouth opening of mean $36.5806+$ $5.2711 \mathrm{~mm}$ ( $p$ value $=0.0000$ significant $)$. As with the control group, showed average mouth opening of 31.4667 $+2.8752 \mathrm{~mm}$ after therapy of ultrasound showed improvement of mouth opening in $39.1333+2.7482 \mathrm{~mm}$.

EMG study done in patients for checking the activity of muscle for pre and post UST changes in muscles are as shown in Figure 4 and Figure 5 respectively.
The distribution according to percentage of relief in muscular activity after Ultrasound therapy for Temporalis, Masseter and Pterygoid muscle is as shown in Figure 6. Spontaneous activity present in Temporalis muscle pre UST period in $73.33 \%$ patients, out of which during post therapy spontaneous activity absent in $60 \%$ patients and activity is reduced in $13.33 \%$. Spontaneous activity present in Masseter muscle pre UST period in $100 \%$ patients, out of which during post therapy spontaneous activity absent in $73.33 \%$ patients and activity reduced in $26.66 \%$ patients. While, spontaneous activity present in Pterygoid muscle pre UST period in $33.33 \%$ patients out of which during post therapy spontaneous activity absent in $26.66 \%$ and activity reduced in $6.66 \%$ patient.

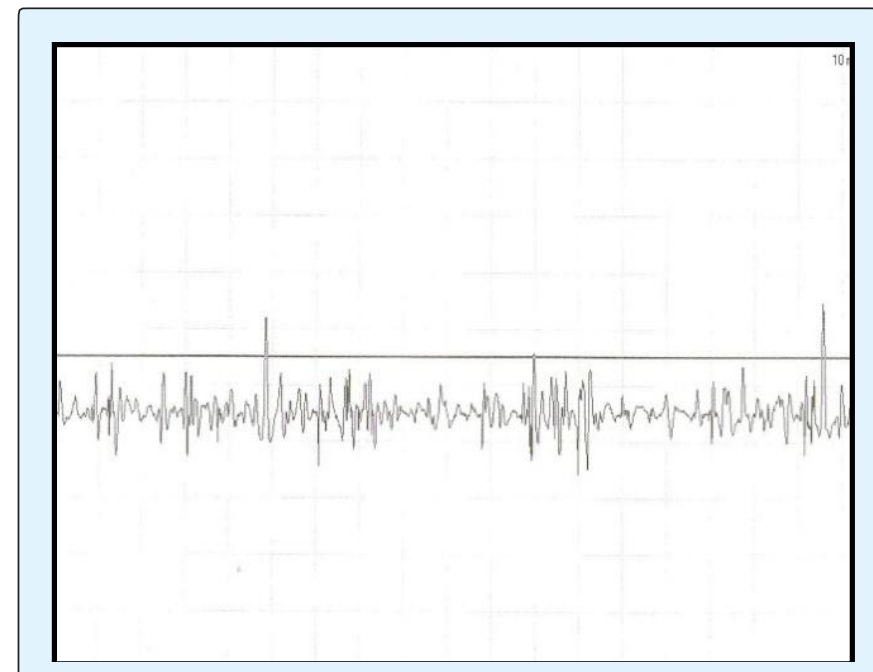

Figure 4: Pre treatment: '++' Activity seen at rest.

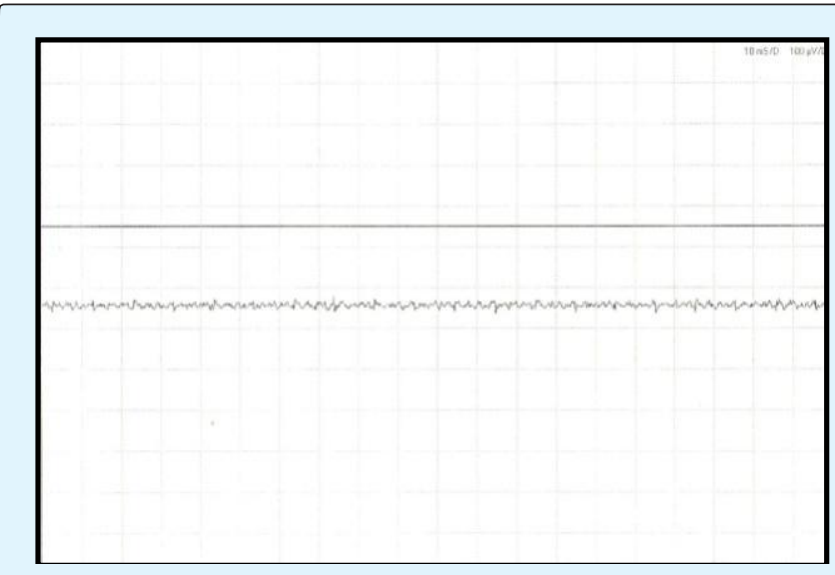

Figure 5: Post Treatment: Sparse activity seen at rest. 


\section{International Journal of Surgery \& Surgical Techniques}

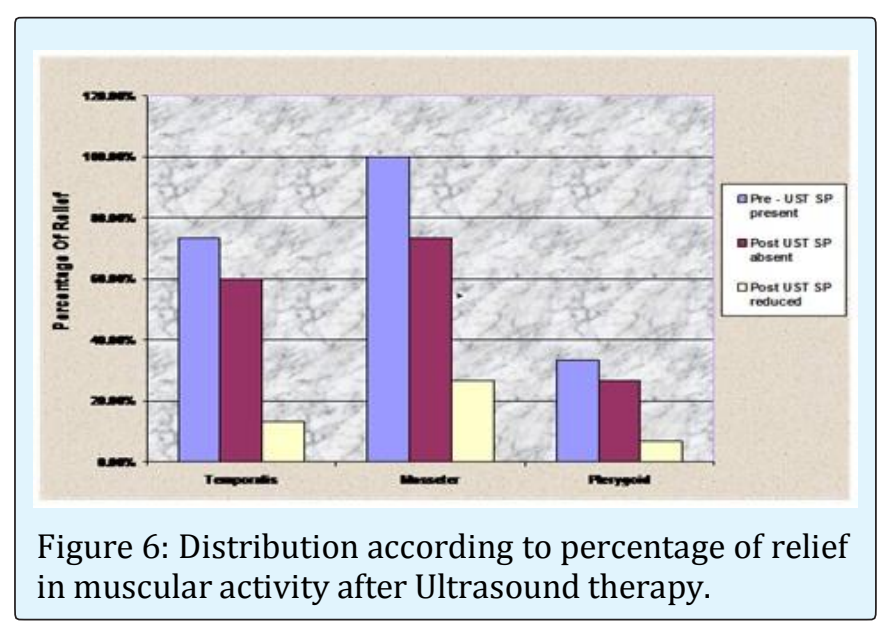

The value of obtaining ultrasound therapy for treating myofascial pain dysfunction syndrome has been stressed by various authors $[3,7,10-13]$.

The study has been carried out to fulfil the aims

a) To evaluate the role of ultrasound therapy in treating the myofascial pain dysfunction syndrome which are of muscular origin

b) To reassure the patient that pain or dysfunction was probably caused by a disturbance of muscular activity and there was probably no disease condition present.

c) To institute physical therapy measures in order to promote bilateral function of jaws

According to Laskin DM [13] women affected MPD syndrome more frequently than men with the ratio in various report ranging from 3:1 to 5:1 although condition can occur in children the greatest incidence appear to in 20- $40 \mathrm{yr}$ age group. Also in study group there is seen predilection to female. As per Laskin DM [13] MPD syndrome believed to be stress related disorder it was hypothesized that increased in muscle tension frequently combine with parafunctional habit. Fine EW [14] did comparative study to evaluate the role of psychologic aspect in MPDS. Also Hidaka 0, et al. [1] proved in 2004, mental stress can cause electromyographic and or hemodynamic changes in jaw muscles thereby inducing jaw muscle pain.

In the present study after psychological assessment it was observed that $15.4 \%$ male patients had anxiety disorder, psychologic factor with medical condition was present in $5.6 \%$ and mood disorder with anxiety was present in $5.6 \%$. Gelb H [15] previously stated that pain associated with TMJ disturbances has been attributed mainly to the masticatory muscle. According to muscular involvement masseter muscle involvement was seen in 93.5\% temporalis muscle involvement seen in $80.6 \%$ pterygoid muscle involvement seen in $19.4 \%$ and muscle involvement of posterior cervical group of muscle was seen in $3.2 \%$.

Laskin DM [13] Ultrasound therapy is considered as the physical therapy recommended for use in patients with MPD syndrome are directed at reducing muscle tension and spasm and improving jaw function. In MPD syndrome the treatment is used over the masseter and temporalis muscle Application last 10- 15 min. Muscle tenderness usually resolve in 1-2 weeks. The electrical stimulation of muscle increases circulation reduces pain and spasm increases resistance to fatigue. While in present study overall improvement seen within 2 weeks. And follow up of patient done for upto $1 \mathrm{yr}$.

\section{The Effect of Ultrasound on Pain Relief $[10,16]$}

Ultrasound induced redirection in the Sodiumpotassium ATP ase pump activity. A decrease in pump activity if it occur in neuronal plasma membranes which inhibit the transudation of noxious stimuli and subsequent neural transmission which may account in part for the pain relief. EMG is only reliable method available for objective recording of a patient muscular function. In this study EMG study done in patients to evaluate the function of muscle changes before and after giving ultrasound therapy and this study proved that UST have profound effect in Myofascial pain dysfunction syndrome particularly in muscular group.

\section{Conclusion}

Ultrasonic therapy provided reveal significant improvement jaw opening and relief of pain within 2 weeks of therapeutic treatment EMG study done to evaluate the changes in muscles of mastication since MPD patients respond to stress with increased masticatory muscle activity spontaneous activity observed in involved muscle but after taking UST the spontaneous activity found absent in most of patients or reduced. Thus ultrasound seems to be an effective physical therapeutic measure for alleviation of discomfort in Myofascial Pain Dysfunction syndrome. If used properly, it is safe, effective and offers a specific type of treatment not duplicated by other form of therapy. The dental profession should be aware of this valuable adjunct to the management of MPDS particularly since dentists are best qualified for comprehensive treatment of this pathologic entity. 


\section{International Journal of Surgery \& Surgical Techniques}

\section{References}

1. Hidaka O, Yanagi M, Takada K (2004) mental stress induced physiological changes in the human masseter muscle. Journal of dental research 83(3): 227-231.

2. Schwartz L and Chayes C M, Facial Pain and Mandibular Dysfunction. Saunder, Philadelphia, pp: 152.

3. Thomson H (1971) Mandibular dysfunction syndrome. British dental journal 130: 187-193.

4. Travell J (1960) TMJ pain referred from muscles of the head and neck. J of Prosth Dent 10: 745.

5. Reading A, Raw M (1976) The treatment of mandibular dysfunction pain. British dental journal 140(6): 201-205.

6. Laskin D M, Block S (1986) Diagnosis and treatment of myofascial pain-dysfunction (MPD) syndrome. Journal of Prosthetic Dentistry 56(1): 75-83.

7. Erickson RI, Sacramento (1964) Ultrasound - a useful adjunct in Temporomandibular joint therapy. Oral Surgery, Oral Medicine and Oral Pathology 18: 176179.

8. Lehmann JF, Biegler R (1954) Changes of Potentials and Temperature Gradients in Membranes Caused by Ultrasound. Arch Phys Med 1954 35(5): 287-295.

9. Widmalm SE, Lee YS, McKay DC (2007) Clinical use of Qualitative Electromyography in the Evaluation of Jaw Muscle function: A practitioner's Guide. Journal of craniomandibular practice 25(1): 63-73.
10. Greene CS, Laskin DM (1983) Long term evaluation of treatment for myofascial pain dysfunction syndrome: A comparative analysis. Journal of American Dental Association 107(2): 235-238.

11. Demmink J H, Helders P J M, Hobaek H, Enwemeka C (2003) the variation of heating depth with therapeutic ultrasound frequency in physiotherapy. Ultrasound in medicine and biology 29(1): 113-118.

12. Elhag M, Coghlan K, Christmas P, Harvey W, Harris M (1985) The anti- inflammatory effect of dexamethasone and therapeutic ultrasound in oral surgery. Br J oral maxillofac surg 23(1): 17-23.

13. Laskin DM, Green CS (1972) Influence of the doctor patient relationship on placebo therapy for patient with myofascial pain dysfunction (MPD) syndrome. Journal of American Dental Association 85(4): 892894.

14. Fine EW (1971) Psychological factors associated with non-organic temporomandibular joint pain dysfunction syndrome. British dental journal 131(9): 402-404.

15. Gelb H, Bernstein I (1983) Clinical evaluation of two hundred patients with Temporomandibular joint syndrome. Journal of prosthetic dentistry 49(2): 234243.

16. Esposito CJ, Veal SJ, Farman AG (1984) Alleviation of myofascial pain with ultrasonic therapy. Journal of prosthetic dentistry 51(1): 106-108.

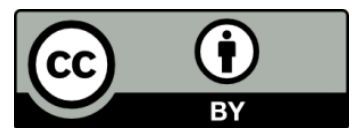

